\title{
Growth and feeding problems after repair of oesophageal atresia
}

\author{
J W L Puntis, D G Ritson, C E Holden, R G Buick
}

\begin{abstract}
Of 230 families belonging to a support group for parents of children born with oesophageal atresia, 124 returned a detailed questionnaire on feeding history and growth. Being slow to feed, refusing meals, coughing or choking during eating, and vomiting at meal times were signficantly more common than in $\mathbf{5 0}$ healthy control children. Anthropometric analysis indicated that almost one third of patients were growth retarded, although those with a primary oesophageal anastomosis were less likely to be stunted or wasted compared with children who had an oesophageal substitution preceded by an oesophagostomy. Feeding problems tended to resolve spontaneously but slowly, with half of all children still reporting some difficulties at 7 years of age. Parents were considerably worried by feed related symptoms and families benefited from mutual support. There is a need for additional help and advice to be provided both by hospital staff interested in nutrition and feeding disorders and those professionals involved with primary care.
\end{abstract}

Tracheo-oesophageal fistula with oesophageal atresia occurs in approximately one in 5000 births. $^{1}$ After surgery there is an overall survival rate approaching $90 \%,{ }^{2}$ and those who reach adulthood can expect to enjoy a normal life. ${ }^{3}$ Despite this, feeding difficulties in early childhood, ${ }^{4}$ and repeated respiratory problems, ${ }^{56}$ contribute to major disruption of family life. ${ }^{7}$ Although it seems that growth in patients reaching adulthood may well be normal, ${ }^{3}$ there is a lack of information concerning growth during childhood and the natural history of feeding problems. Feeding difficulties severe enough to affect nutritional state might be expected to influence prognosis in some children. The aims of this study were to determine the prevalence of certain feeding related symptoms in children who have undergone surgery for oesophageal atresia, to explore how parents coped, and to investigate any possible impact on growth.

Patients and methods

A detailed questionnaire relating to feeding behaviour was sent to all 230 families belonging to the Tracheo-Oesophageal Fistula Support Group (TOFS), an organisation set up in the United Kingdom in 1982 by parents of children born with oesophageal atresia and tracheooesophageal fistula in order to share information
Calculation of feeding score ${ }^{\star}$

These questions were asked in relation to both milk feeds and introduction of solid foods:

Did your child take a long time to finish a feed?

Did your child refuse to feed at times?

Did your child experience coughing or choking during feed times?

Did your child vomit during or after feeds?

Parents were asked to indicate for each question whether symptoms occurred with (score):

$\begin{array}{ll}\text { All feeds } & 6 \\ \text { Most feeds } & 5 \\ \text { Half of feeds } & 4 \\ \text { 1-2 Feeds/day } & 3 \\ \text { 1-2 Feeds/week } & 2 \\ \text { Occasional } & 1 \\ \text { None } & 0\end{array}$

${ }^{\star}$ Maximum possible score 48

and advice. Details of early feeding history were requested and parents asked to indicate the frequency with which specified feed related symptoms had occurred (table). Whether or not, and at what age, symptoms had finally resolved were also recorded. This part of the questionnaire was administered to parents of 50 healthy controls who were either children of colleagues, or siblings of children in hospital. The answers to each question were scored according to frequency of symptom (table) and a 'feeding score' calculated for each child in an attempt to quantify feeding difficulty. Parents were asked to provide accurate height and weight measurements and information regarding whether or not an oesophagostomy had been performed. Height for age, and weight for height standard deviation (SD) scores were calculated for all patients in whom height and weight data were recorded. ${ }^{8}$ Finally, part of the questionnaire was left unstructured for parents to outline their management of feeding difficulties.

\section{STATISTICAL EVALUATION}

Feeding scores for different groups of patients and controls were compared using the MannWhitney U test. Height for age, and weight for height SD scores were compared with the reference population mean using Student's $t$ test. Other comparisons between groups were made using the $\chi^{2}$ test and Fisher exact probability test. Correlations were calculated using Spearman's method.

\section{Results}

A total of 124 of the 230 questionnaires were returned (male:female 2:1). Ninety two (74\%) 
of the children with a median age of 4.3 years (range $0.5-22.8$ ) had undergone a primary oesophageal anastomosis. Thirty two (26\%) initially had an oesophagostomy and gastrostomy, later followed by an oesophageal substitution procedure. The age range in this group was similar to the primary anastomosis group: mean age 5.6 years (range $1 \cdot 3$ to $22 \cdot 3 ; \mathrm{p}=0 \cdot 3$ ). The median age at closure of oesophagostomy was 1 year (range $0 \cdot 1$ to $2 \cdot 5$ ). Fifty replies were obtained from control children, median age 4.5 years (range 0.6 to 12.0 ).

\section{FEEDING IN HOSPITAL}

\section{Primary anastomosis group}

Half the parents reported that nursing staff always allowed them to give feeds when they visited hospital but $10(11 \%)$ were never permitted to do so. Forty six mothers $(50 \%)$ breast fed their infants for a median duration of three months (range two weeks to 21 months). Fourteen (15\%) were discouraged from attempting to breast feed and only two rejected this advice.

\section{Oesophagostomy group}

Two thirds of parents were always permitted to give feeds when they visited hospital and only two $(6 \%)$ never allowed. Among the 32 children with an oesophagostomy, all but five had sham feeds at most feed times and only one patient was never sham fed. Compared with the primary anastomosis group, significantly more mothers of children in the oesophagostomy group (11 (34\%); $p=0.02)$ felt that they were discouraged from breast feeding. Despite this, a similar proportion $(12,38 \%)$ did breast feed. The median duration of breast feeding was 2.5 months (range one week to seven months), which was not significantly different from the primary anastomosis group.

Insufficient milk supply was the most common reason for giving up breast feeding. No mother reported inherent difficulties in feeding a child with an oesophageal repair or oesophagostomy as the reason for stopping.

\section{General advice}

A minority (45\%) of parents were warned before hospital discharge about possible feeding problems and only one third of these received specific advice on management of meal times. Ward nurses were the most frequent source of advice, followed by doctors. Most commonly mentioned was the possibility of choking during feeds, and that feeds were best given slowly. However, only one parent reported being told what to do should this problem occur. Eight (6\%) parents discussed feeding with a hospital dietitian.

SPECIFIC FEED RELATED DIFFICULTIES

The median age at introduction of solid food was 4 months (range 2 to 7 months) and for solid food containing lumps, 8 months (range 5 to 16 months). The following feed related symptoms were reported to occur with two or more milk feeds a day: slow to feed, six (12\%) children; coughing or choking with feed, three $(6 \%)$; vomiting, four $(8 \%)$; and feed refusal, five $(10 \%)$. The prevalence of these symptoms in relation to introduction of solid feeds was considerably reduced, being $4 \%, 2 \%, 2 \%$, and $4 \%$ respectively. By 1 year of age, all symptoms had resolved in 37 (74\%) children, and $45(90 \%)$ children aged 2 or more years were completely symptom free.

\section{Primary anastomosis group}

The median age at introduction of solid foods was 6 months (range 1.5 to 30 months) and solids containing lumps, 12 months (range 2 to 54 months). The introduction of solids was significantly delayed compared with the control group $(p<0.0001)$. During two or more milk feeds each day, over half the children $56(61 \%)$ were considered by parents to be slow to feed, almost one third $(29,32 \%)$ had episodes of choking or coughing, $16(17 \%)$ vomiting, and $18(20 \%)$ feed refusal. The numbers were similar during introduction of solids: $48(52 \%)$ were slow to feed, one third $(29,32 \%)$ experienced coughing or choking, $15(16 \%)$ vomiting, and $15(16 \%)$ refused to take feeds. Compared with the control group the prevalence of milk feed refusal or vomiting was not significantly different $(p>0 \cdot 1)$ while being slow with feeds $(p<0.001)$ and coughing or choking with milk $(p=0.0002)$ were much more common. All problems related to solid foods were significantly more common than in the control group: food refusal $(p=0.02)$, taking a long time to feed $(p<0.0001)$, coughing and choking $(p<0.0001)$, and vomiting $(p=0.007)$. Of the 88 children followed up for one year, only $18(20 \%)$ were free of these feed related symptoms, while among 21 children followed up for seven years, $12(57 \%)$ were symptom free.

\section{Oesophagostomy group}

The median age at introduction of solid food was 12 months (range 3 to 24 months), significantly later than in the control group $(p=0.0003)$, and solid food with lumps 18 months (range 9 to 48 months). Both blended solids and solids with lumps were introduced significantly later than in the primary anastomosis group $(p=0.001$; $p=0.04$ ). The frequency with which specific feed related symptoms occurred (after closure of oesophagostomy) during at least two feeds each day were similar to those seen in the primary anastomosis group. For milk feeds the figures were: $12(38 \%)$ slow to feed, six (19\%) coughing or choking, $10(31 \%)$ vomiting, and seven $(22 \%)$ feed refusal. Significantly fewer children were slow to take milk feeds $(p<0.05)$ than in the primary anastomosis group. For solid feeds the proportion of children with feed related symptoms were: $16(50 \%)$ slow to feed, nine $(28 \%)$ choking, 10 (31\%) vomiting, and six (19\%) refusal. Comparing symptoms during milk 
feeds with the control group, feed refusal and coughing were not significantly different, though taking a long time to feed and vomiting were both more common after oesophagostomy $(\mathrm{p}<0.01)$. All symptoms related to solid feeds were significantly more common than among controls: food refusal $(p=0 \cdot 04)$, slow with meals $(\mathrm{p}<0.0001)$, coughing and choking $(\mathrm{p}=0.0007)$, and vomiting $(p=0.0002)$. At 1 year of age no children were completely free of these feed related symptoms, while eight $(66 \%)$ out of 12 followed up for seven years were asymptomatic.

\section{Troublesome foods}

Difficulty swallowing certain types of foods was reported by $24(26 \%)$ of the primary anastomosis group compared with two $(6 \%)$ of the oesophagostomy group $(\mathrm{p}<0.01)$. Many other children were avoiding particular foods for the same reason. The most frequent offenders were similar in each group: meat $(37 \%$ patients), apple $(23 \%)$, bread $(23 \%)$, oranges (14\%), and raw vegetables (12\%). A further 38 types of food were listed but many items only by one or two families.

\section{GROWTH}

Primary anastomosis group

Height and weight data were provided for 73 children of whom 10 were stunted (height for age $>2$ SDs below the mean), seven wasted (weight for height $>2$ SDs below the mean), and two both stunted and wasted. The mean (SD) height for age SD score was $-0.5(1.8)$ and mean (SD) weight for height SD score -0.4 $(1 \cdot 6)$. The group was therefore significantly growth retarded compared with normal population standards for both height for age and weight for height $(p=0.02 ; p=0.04)$.

\section{Oesophagostomy group}

Anthropometric data was provided for 27 children in this group of whom nine were stunted, two wasted, and two both stunted and wasted; this is a significantly higher proportion than in the primary anastomosis group $(p<0.05)$. The mean (SD) height for age SD score was $-1.78(1.7)$ and the mean (SD) weight for height SD score $-1 \cdot 1(0 \cdot 9)$; both were significantly below the normal population $(p<0.0001)$ and the primary anastomosis group $(p=0.002 ; p=0.01)$. The prevalence of growth retardation did not decrease with increasing age in either group.

\section{FEEDING SCORE}

The median feeding score for the primary anastomosis group was 16 (range 0 to 42 ), and for the oesophagostomy group 13 (0 to 48$)$. No significant differences in scores were found comparing either each group, or growth retarded, with normally grown children. Feeding scores did not significantly correlate with height for age or weight for height SD scores.
OESOPHAGEAL DILATATIONS

Among the primary anastomosis group 48 patients had been admitted to hospital for elective oesophageal dilatation while 38 had been admitted as emergencies with food stuck in the oesophagus. From the oesophagostomy group 12 underwent elective dilatations of the oesophageal anastomosis and 10 were admitted for emergency oesophagoscopy. Of the patients admitted as emergencies, five were found to have impacted foreign bodies, and food residue were retrieved from the others. Meat was the most common $(n=20)$, followed by apple $(n=10)$, carrot $(n=7)$, and sausage $(n=6)$. The overall proportion of patients in each group having oesophageal dilatations was not significantly different $(\mathrm{p}>0.05)$.

\section{SOURCES OF ADVICE FOR PARENTS}

Only $14(11 \%)$ of parents reported discussing feeding problems with hospital medical, surgical, and nursing staff; most turned to friends, other members of TOFS, general practitioner, or health visitor. One third of parents had discussed feeding problems during follow up with a hospital dietitian, although a further $57(46 \%)$ indicated that they would have liked to have had this opportunity. Hospital staff were the source of information regarding the existence of the support group for $46(37 \%)$ of parents.

\section{MANAGEMENT OF FEEDING PROBLEMS \\ BY PARENTS}

Some parents stated that feeding difficulties dominated their lives and many expressed feelings of isolation and helplessness. The importance of patience, coupled with optimism about the final outcome, was often emphasised.

Sham feeding and gastrostomy feeds

Sham feeding was regarded as an important way of facilitating the return to normal feeding once oesophageal substitution was performed. Almost half of families considered the extra cost to be signficant and four had successfully applied to the Department of Health and Social Security for assistance. One parent had designed a stand to which the gastrostomy feed dispenser could be attached leaving both hands free to sham feed the baby. An adjustable gate clamp on the gastrostomy tube regulates the flow of milk. Sham feeding can be a messy business and a towelling nappy or absorbent paper padding around the neck was used to protect the skin; stoma nurses had been useful sources of advice regarding skin care for some patients. Flushing the gastrostomy tube with water after a feed helped prevent blocking and securing the spiggot in place with sticky tape can prevent it from becoming dislodged and allowing reflux of stomach contents up the tube. Tucking the gastrostomy tube back into the babies clothing prevents its accidental removal; 'all in one' towelling romper suits are useful garments in this respect. 


\section{Mealtimes}

Thirty two percent of parents commented that a food blender was an essential item of equipment; $60(48 \%)$ stressed the importance of keeping babies relatively upright when feeding. Taking time over meals and not attempting to force feed a child were seen as most important, together with the inclusion of the child within the family group at meal times. The necessity of variety in diet was also emphasised. Initially, solid foods were liquidised and lumpy food gradually introduced progressing from finely minced, coarsely minced, to increasingly large lumps. Casseroles or other dishes with plenty of juices rather than dry foods were preferred. Some parents found confectionary (for example, white chocolate) or savouries that melt in the mouth (for example, 'Wotsits') useful for teaching their child to take solids without provoking symptoms.

\section{Choking}

Hurrying over food was regarded as a frequent antecedent of choking and a liberal fluid intake during meals as preventative. In the event of acute dysphagia or choking, parents advocated staying calm and back slapping, giving a drink, or inducing vomiting as means of dislodging food. Once the choking episode was over and the child fully recovered it was important to carry on with the rest of the meal.

\section{Discussion}

Despite the fact that parents of symptomatic patients are probably more likely to join a support group than those of asymptomatic children, this study has confirmed that feeding difficulties are a major problem after repair of oesophageal atresia. Approximately one third of patients in our survey had severe symptoms such as coughing, choking, vomiting, and refusal at least twice a day in the first year of life, significantly more than control subjects. While the proportion of symptom free patients increased with length of follow up (after seven years two thirds of patients in both groups were symptom free), nearly one third of children over the age of 7 continued to report symptoms, principally dysphagia associated with certain types of food. Initially these symptoms were a source of great concern to parents and had considerable impact on family life.

In addition, anthropometric data reported by parents suggested a worryingly high prevalence of growth failure (32 out of 100 patients for whom height and weight data were provided) with both groups being significantly below the reference population means for the height for age and weight for height. Only six children had other medical conditions that might have contributed to growth failure. Whereas parental reporting of height and weight is unlikely to be accurate, other studies have suggested a similar prevalence of growth failure after repair of oesophageal atresia. ${ }^{2-11}$ The oesophagostomy patients were significantly less well grown than the primary anastomosis group (height for age $p=0.002$, weight for height $p=0.01$, which may reflect greater periods of time in hospital or more refractory feeding difficulties. We did not find a decrease in the proportion of growth retardation with increasing age, suggesting that poor feeding (or whatever other factors might be adversely affecting nutritional state) may have long term effects on growth.

Failure to find a significant negative correlation between 'feed scores' and indicators of nutritional state may reflect both inaccuracy of parental height and weight reporting and the fact that the 'feed score' gives a static measurement of feeding difficulties in relation to introduction of milk and solid feeds and does not take into account overall duration of symptoms.

As an indicator of malnutrition growth requires careful monitoring during follow up. ${ }^{12}$ In a recent long term study of patients born with oesophageal atresia it is encouraging that all were found to be normally grown adults. ${ }^{3}$ Follow up information was available for only $50 \%$ of patients operated on over a given period, however, and, as chronic malnutrition and growth failure may affect mortality, nutritionally compromised patients might have been among the $50 \%$ who either did not survive childhood or were lost to follow up for other reasons.

Parents evidently find difficulty in dealing with feeding problems and yet may be offered little useful advice. ${ }^{4}$ Breast feeding need not be discouraged, and before discharge from hospital, feeding problems and their management should be fully discussed. A timetable for weaning should be formulated including a plan for graded introduction of different consistencies of solid foods over a period of several months. The fact that each child is different should be highlighted as some have minimal or no difficulties. Although such discussion may take place between parents and nursing or medical staff, dietitians should be involved when possible. During follow up only a third of parents were able to meet a dietitian, although over half the remainder indicated that they would have taken advantage of such contact if offered. Consultation with a dietitian might also help reduce the proportion of parents (31\%) dissatisfied with the diets provided on the ward during periods of admission to hospital.

Food 'sticking' can be the result of disordered oesophageal motility and will clear spontaneously or after a drink. Persistent and frequent symptoms should raise the possibility of a stricture and oesophagostomy may be necessary for full assessment. Routine oesophagoscopy and anastomotic dilatation is unnecessary in many children. Choking probably results from aspiration either secondary to disordered oesophageal motility, ${ }^{13}$ or after a food bolus has become lodged. Asphyxia is therefore unlikely to occur and reassurance together with a drink will be all that is required. Choking and vomiting can also be indicative of gastro-oesophageal reflux, which is common after repair of oesophageal atresia and sometimes requires antireflux surgery. ${ }^{14}$ Oesophagitis associated with gastro-oesophageal reflux can lead to food refusal or reluctance to feed and should then be suspected even in the absence of vomiting. ${ }^{15}$ Twenty four hour 
oesophageal $\mathrm{pH}$ monitoring is a valuable diagnostic tool in this context and may also help predict the need for further surgery. ${ }^{16}$

Feeding problems can come to dominate the lives of parents who may feel particularly helpless when denied one of their important parenting roles by a child unable or unwilling to eat. Whereas optimism in the long term is justified, support and encouragement are required in the early stages. This study indicates areas of management that require closer attention by staff based both in hospital and the community, and, through borrowing from the experiences of parents themselves provides practical advice for those caring for children who have undergone surgery for oesophageal atresia and tracheo-oesophageal fistula.

We would like to thank members of TOFS for their help and participation in this study and Mrs Linda Morris for her support and encouragement.

1 Ein SH, Friedberg J. Esophageal atresia and tracheoesophageal fistula: review and update. Otolaryngol Clin North Am 1981;14:219-49.

2 Spitz L, Kiely E, Brereton RJ. Oesophageal atresia: five year experience with 148 cases. $\mathcal{f}$ Pediatr Surg 1987;22:103-8.

3 Chetcuti P, Myers NA, Phelan PD, Beasley SW. Adults wh survived repair of congenital oesophageal atresia and tracheo-oesophageal fistula. Br Med 7 1988;297:344-6.
4 Smith IJ, Beck J. Mechanical feeding difficulties after primary repair of oesophageal atresia. Acta Paediatr Scand 1985;74:237-9.

5 Shermeta DW, Whitington PF, Seto DS, Haller JA. Lower esophageal sphincter dysfunction in esophageal atresia: nocturnal regurgitation and aspiration pneumonia. $f$ Pediatr nocturnal regurgitation $1977 ; 12: 871-6$.

6 Dudley NE, Phelan PD. Respiratory complications in longterm survivors of oesophageal atresia. Arch Dis Child term survivors

7 Koop CE, Schnaufer L. Thompson G, Haecker T, Dalrymple $\mathrm{D}$. The social, psychological and economic problems of the patient's family after successful repair of esophageal atresia. $Z$ Kinderchir 1975;17:125-31

8 World Health Organisation. Measuring change in nutrition status. Geneva: WHO, 1983.

9 Laks H, Wilkinson RH, Schuster SR. Long-term results following correction of esophageal atresia with tracheoesophageal fistula: a clinical and cinefluorographic study. F Pediatr Surg 1972;7:591-7.

10 Andrassy RJ, Patterson RS, Ashley J, Patrissi G, Hossein Mahour G. Long-term nutritional assessment of patients with esophageal atresia and/or tracheoesophageal fistula. f Pediatr Surg 1983;18:431-5.

11 Gutierrez-Sanroman C, Vila-Carbo JJ, Segarra-Llido V, Garcia-Sala C, Ruiz-Company S. Long-term nutritional evaluation of 70 patients operated on for esophageal atresia. Pediatric Surgery International 1988;3:123-7.

12 Tanner JM. Growth as a monitor of nutritional status. Proc Nutr Soc 1976;35:315-22.

13 Orringer MB, Kirsh MM, Sloan H. Long-term esophageal Orringer MB, Kirsh $M M$, Sloan $\mathrm{H}$. Long-term esophageal
function following repair of esophageal atresia. Ann Surg

14 Parker AF, Christie DL, Cahill JL. Incidence and significance of gastroesophageal reflux following repair of esophageal atresia and tracheoesophageal fistula and the need for anti-reflux procedures. F Pediatr Surg 1979;14:5-8. 15 Paton JY, Nanayakkhara CS, Simpson H. Vomiting and gastro-oesophageal reflux. Arch Dis Child 1988;63:837-8.

16 Evans DF, Haynes J, Jones JA, Stower MJ, Kapila L. Ambulatory esophageal $\mathrm{pH}$ monitoring in children as an indicator for surgery. $\mathcal{F}$ Pediatr Surg 1986;21:221-3. 